\title{
Proposed Methods for Real-Time Measurement of Posterior Condylar Angle during TKA
}

\author{
Prateek Behera, $\mathrm{MS}^{1}$, Devendra Kumar Chouhan, $\mathrm{MS}^{1}$, Mahesh Prakash, $\mathrm{MD}^{2}$, and Mandeep Dhillon, $\mathrm{MS}^{1}$ \\ Departments of ${ }^{1}$ Orthopedics and ${ }^{2}$ Radiodiagnosis, Postgraduate Institute of Medical Education and Research, Chandigarh, India
}

\begin{abstract}
Purpose: Conventional instruments are known to result in high numbers of outliers in restoring femoral component rotation primarily due to fixed degree of external rotation resection relative to the posterior condylar line (PCL). Outliers can be reduced by determining the patient specific posterior condylar angle (PCA) preoperatively or intraoperatively. There is a paucity of methods that can be used during surgery for determining the PCA. We propose two simple, real-time methods to determine the PCA and hence to measure the axial anatomical variation during surgery.

Materials and Methods: The study was conducted using axial computed tomography (CT) scans of the knees of 26 patients. The commercial software K-PACS and our proposed two methods (trigonometric and protractor) were used to measure the angle between the transepicondylar axis and PCL, i.e., PCA. Statistical comparison between the mean angles obtained by K-PACS and our methods were done.

Results: The three methods resulted in similar PCAs. The mean PCA measured by the three methods were similar. The mean PCA value measured by the K-PACS, trigonometric method and protractor method was $6.27^{\circ}$ (range, $0^{\circ}$ to $12^{\circ}$ ), $6.23^{\circ}$ (range, $0^{\circ}$ to $11.11^{\circ}$ ) and $6.31^{\circ}\left(\right.$ range, $0^{\circ}$ to $12^{\circ}$ ), respectively. There were significant correlations between the K-PACS measured PCA and trigonometrically or protractor measured PCA.

Conclusions: Our novel, simple, easily reproducible, real-time and radiation-free PCA measurement methods obviate the need for preoperative CT scan for identification of patient specific PCA.
\end{abstract}

Keywords: Knee, Osteoarthritis, Arthroplasty, Posterior condylar axis

\section{Introduction}

Longevity and outcome of total knee arthroplasty (TKA) are dependent on the restoration of mechanical axis and ligamentous balance in both flexion and extension ${ }^{1,2)}$. To improve the accuracy of alignment and clinical outcomes, technologies such as computer-assisted surgery, patient matched instrumentation and robotic surgery are increasingly being used ${ }^{3}$. Although many studies have found superiority of these new technologies in

Received May 19, 2014; Revised (1st) July 27, 2014;

(2nd) September 3, 2014; (3rd) September 12, 2014;

Accepted September 16, 2014

Correspondence to: Devendra Kumar Chouhan, MS

Department of Orthopedics, Postgraduate Institute of Medical

Education and Research, Chandigarh 160012, India

Tel: +91(887)201-6154, Fax: +91-172-2744401

E-mail: drdevnim@gmail.com

This is an Open Access article distributed under the terms of the Creative Commons Attribution Non-Commercial License (http://creativecommons.org/licenses/by-nc/3.0/) which permits unrestricted non-commercial use, distribution, and reproduction in any medium, provided the original work is properly cited. achieving better alignment compared to the conventional methods, it still remains questionable whether these improvements result in better clinical outcomes ${ }^{3}$. These technologies increase the $\operatorname{cost}^{3,4}$, and cost effectiveness studies have not demonstrated their superiority over the conventional instrumentation ${ }^{4)}$.

Limitation of conventional instrumentation in restoration of correct femoral component rotation ${ }^{5}$ has been considered as the commonest culprit for abnormal patellofemroal kinematics and patient dissatisfaction ${ }^{6-8)}$. This limitation of conventional instrumentation is probably attributable to flexion resection in a fixed degree of external rotation to the posterior condylar line (PCL). Studies have identified that the posterior condylar angle (PCA) is not constant in all patients and it varies according to individuals and the pathology affecting the knee ${ }^{9}$. Thus, if we know the patient specific PCA, then the use of an appropriate flexion cutting jig could reduce the number of outliers. Preoperative or intraoperative computed tomography (CT) scan was advocated to identify patient specific axial distal femoral geometry ${ }^{10}$. However, use of CT scan adds to the cost and there are availability issues. In addition, CT scan-based methods are not considered real time methods as they do not take cartilage thickness into account 
while measuring the $\mathrm{PCA}^{11}$.

In this article, we propose simple, easily reproducible, real-time and radiation-free methods to identify patient specific PCA during TKA. We tried to compare the accuracy of our methods with the currently available gold standard tool, i.e., CT scan ${ }^{11}$.

\section{Materials and Methods}

CT scans of 26 patients were randomly selected from our departmental database irrespective of their primary complaints. All the necessary measurements were done on the axial CT images using K-PACS software (www.k-pacs.net, Dr.med.Andreas Knopke, Germany) by a single investigator. We measured the

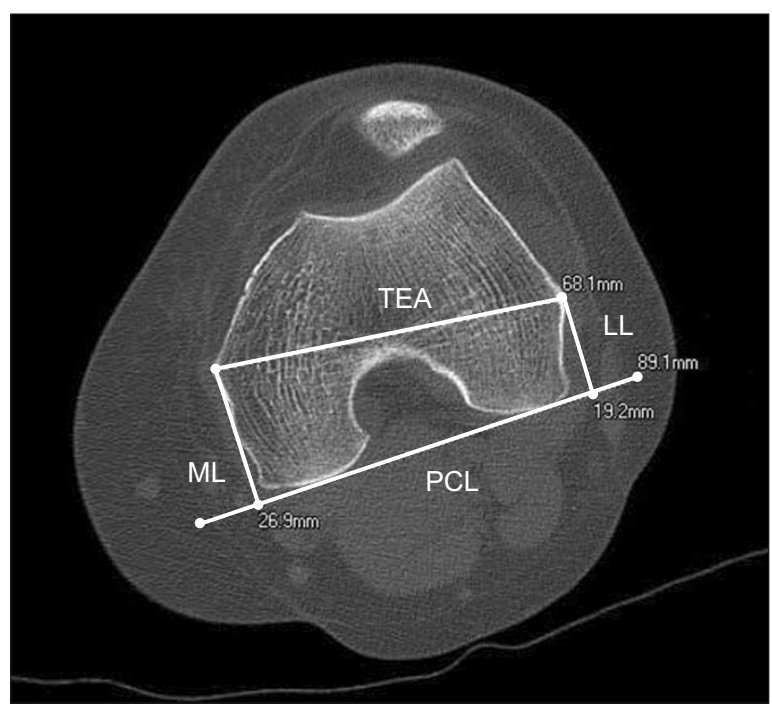

Fig. 1. Measurements using K-PACS. ML: medial line, LL: lateral line, TEA: transepicondylar axis, PCL: posterior condylar line.

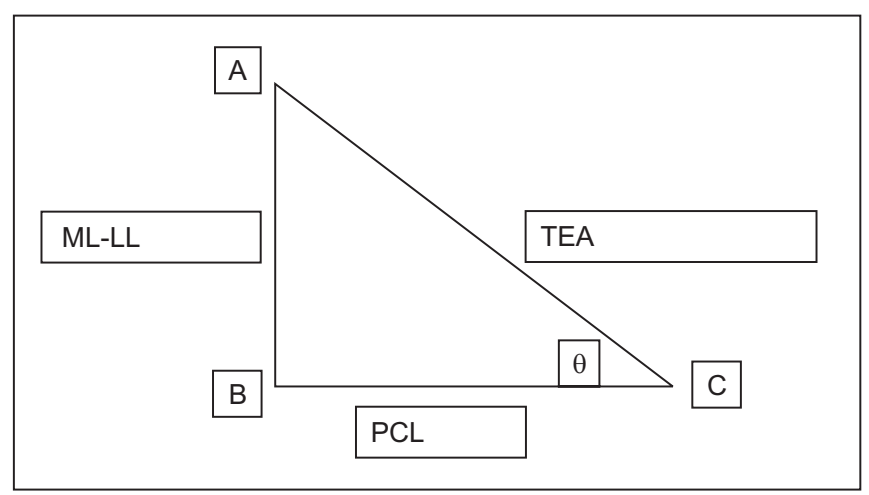

Fig. 2. Method-1: trigonometric method for posterior condylar angle calculation. The trigonometric formula was used to calculate the $\theta$ angle: $\theta=\operatorname{Tan}^{-1}(\mathrm{AB} / \mathrm{BC})$. ML: medial line, LL: lateral line, TEA: transepicondylar axis, PCL: posterior condylar line. angle between the clinical transepicondylar axis (TEA, the line joining the most prominent points of the medial and lateral epicondyles) and the PCL by using the software's algorithms as shown in Fig. 1. In order to implement our method, we measured the distance between both epicondyles, i.e., the length of the TEA and also the lengths of the perpendicular lines connecting lateral and medial epicondyles to PCL; these perpendicular lines were respectively named the lateral line (LL) and medial line (ML) (Fig. $1)$.

\section{Trigonometrically Measured PCA}

The measurements (length of TEA, LL, and ML) obtained by the K-PACS were used to construct a right angled triangle with the angle between TEA and PCL (the angle $\theta$ ) representing the PCA. This angle can be calculated by trigonometric formula, $\theta=\tan ^{-1}(\mathrm{AB} / \mathrm{BC})$ as described in Fig. 2 .

\section{Protractor Measured PCA}

We drew a trapezoid (Fig. 3) based on the previously obtained measurements on a paper and then subtracted a rectangle out of it, and hence left with a right angled triangle. The angle $\theta$ at the base of this right angled triangle was measured with a protractor.

All values obtained by the three methods were analysed using the SPSS ver. 20 (IBM Corp., Armonk, NY, USA). We used paired samples $t$-test for comparison of the mean measurement values of the different methods. Comparisons between K-PACS measured PCA and trigonometrically measured PCA and between

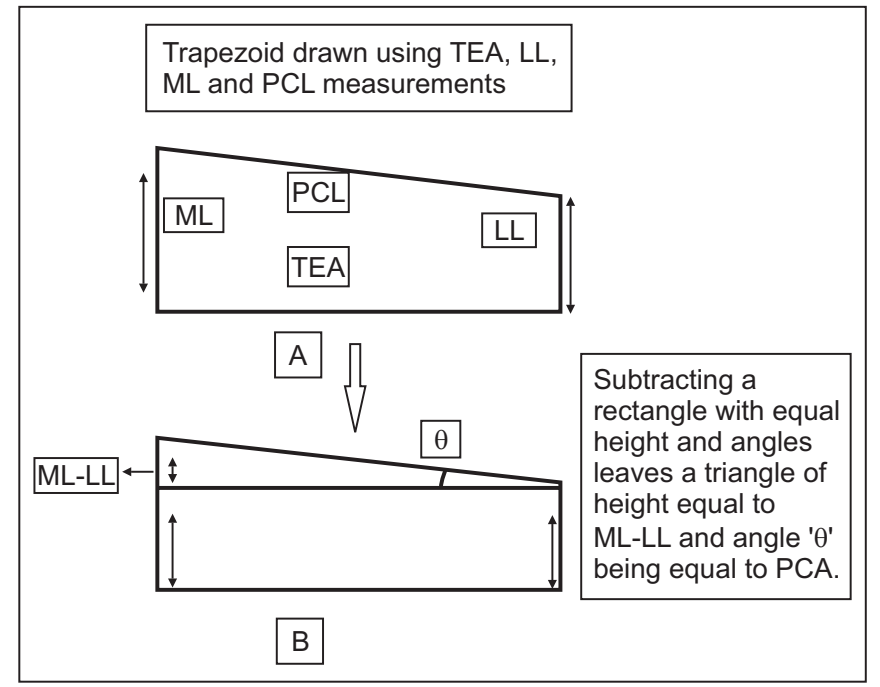

Fig. 3. Method-2: protractor method for PCA measurement by drawing a trapezoid using TEA, ML, LL and connecting PCL. PCA: posterior condylar angle, TEA: transepicondylar axis, ML: medial line, LL: lateral line, PCL: posterior condylar line. 
the K-PACS measured PCA and protractor measured PCA were done. An intraclass correlation coefficient (ICC) analysis was also performed. A p-value of $<0.05$ was considered statistically significant.

\section{Results}

The data collected were the angle measured by K-PACS, base length TEA, LL, and ML. These measurements were applied to obtain the trigonometrically measured PCA (method 1) and protractor measured PCA (method 2), which are summarized in Table 1.

The mean value of the angle between the PCL and the clinical TEA, i.e., PCA, measured by the K-PACS was $6.27^{\circ}$ (range, $0^{\circ}$ to $12^{\circ}$ ) with a standard deviation of $2.97^{\circ}$. The mean value of trigonometrically measured PCA was $6.23^{\circ}$ (range, $0^{\circ}$ to $11.11^{\circ}$ ) with a standard deviation of $2.90^{\circ}$ and of protractor measured PCA was $6.31^{\circ}$ (range, $0^{\circ}$ to $12^{\circ}$ ) with a standard deviation of $2.85^{\circ}$. We analysed the mean PCAs obtained by the three methods using the paired samples t-test. The null hypothesis for statistical analysis was that there was no statistically significant difference between the K-PACS measured PCA and trigonometrically measured PCA (pair 1) and between the K-PACS measured PCA and protractor measured PCA (pair 2). The p-values were found to be 0.726 and 0.746 for pair 1 and 2 , respectively. The p-value was $>0.05$ and the null hypothesis was accepted as true in each case, and hence there was no statistically significant difference between the measurements by the proposed methods and by the K-PACS.

Table 1. Summary of PCA Measurements by the Three Different Methods

\begin{tabular}{|c|c|c|c|c|c|c|}
\hline Case & $\begin{array}{c}\text { K-PACS } \\
\text { measured PCA }\left({ }^{\circ}\right)\end{array}$ & $\mathrm{LL}(\mathrm{mm})$ & $\mathrm{ML}(\mathrm{mm})$ & Base length (mm) & $\begin{array}{l}\text { Trigonometrically } \\
\text { measured PCA }\left({ }^{\circ}\right)\end{array}$ & $\begin{array}{c}\text { Protractor } \\
\text { measured PCA }\left({ }^{\circ}\right)\end{array}$ \\
\hline 1 & 6 & 19.1 & 24.6 & 65.9 & 4.77 & 5 \\
\hline 2 & 6 & 16.7 & 23.1 & 64.9 & 5.63 & 6 \\
\hline 3 & 2 & 25.1 & 27.5 & 71.8 & 1.91 & 3 \\
\hline 4 & 4 & 20.2 & 26.0 & 67.6 & 4.9 & 5 \\
\hline 5 & 10 & 17.0 & 31.2 & 77.5 & 10.3 & 10 \\
\hline 6 & 6 & 19.8 & 27.1 & 67.5 & 6.17 & 6 \\
\hline 7 & 12 & 13.0 & 26.2 & 67.2 & 11.11 & 11 \\
\hline 8 & 4 & 23.0 & 27.2 & 72.2 & 3.33 & 4 \\
\hline 9 & 5 & 20.0 & 27.1 & 68.4 & 5.92 & 6 \\
\hline 10 & 3 & 25.6 & 29.9 & 80.8 & 3.04 & 3 \\
\hline 11 & 5 & 27.1 & 34.5 & 86.6 & 4.88 & 5 \\
\hline 12 & 9 & 19.5 & 32.5 & 77.4 & 9.53 & 10 \\
\hline 13 & 9 & 19.8 & 29.9 & 67.2 & 8.55 & 9 \\
\hline 14 & 6 & 17.6 & 24.4 & 69.0 & 5.63 & 6 \\
\hline 15 & 9 & 16.6 & 28.6 & 71.9 & 9.47 & 9 \\
\hline 16 & 8 & 21.5 & 32.1 & 80.8 & 7.47 & 7 \\
\hline 17 & 0 & 21.7 & 21.7 & 72.1 & 0 & 0 \\
\hline 18 & 8 & 18.2 & 28.0 & 71.8 & 7.77 & 8 \\
\hline 19 & 1 & 21.5 & 23.8 & 69.0 & 1.9 & 1 \\
\hline 20 & 6 & 21.5 & 30.2 & 68.1 & 7.28 & 7 \\
\hline 21 & 10 & 17.2 & 30.6 & 73.7 & 10.3 & 10 \\
\hline 22 & 3 & 24.3 & 28.7 & 86.8 & 2.9 & 3 \\
\hline 23 & 7 & 18.5 & 25.9 & 70.4 & 6 & 6 \\
\hline 24 & 8 & 17.8 & 27.4 & 64.8 & 8.15 & 8 \\
\hline 25 & 9 & 14.8 & 24.6 & 64.7 & 8.61 & 9 \\
\hline 26 & 7 & 19.3 & 27.4 & 72.9 & 6.34 & 7 \\
\hline
\end{tabular}

PCA: posterior condylar angle, LL: lateral length, ML: medial length. 
Table 2. ICC between K-PACS Measured PCA and Trigonometrically Measured PCA

\begin{tabular}{|c|c|c|c|c|c|c|c|}
\hline \multirow{2}{*}{ Measure } & \multirow{2}{*}{ ICC } & \multicolumn{2}{|c|}{$95 \% \mathrm{CI}$} & \multicolumn{4}{|c|}{ F test with true value } \\
\hline & & Lower bound & Upper bound & Value & df1 & df2 & p-value \\
\hline Single & 0.982 & 0.966 & 0.991 & 160.860 & 25 & 50 & 0.000 \\
\hline Average & 0.994 & 0.988 & 0.997 & 160.860 & 25 & 50 & 0.000 \\
\hline
\end{tabular}

ICC: intraclass correlation coefficient, PCA: posterior condylar angle, CI: confidence interval.

Table 3. ICC between K-PACS Measured PCA and Trigonometrically Measured PCA

\begin{tabular}{|c|c|c|c|c|c|c|c|}
\hline \multirow{2}{*}{ Measure } & \multirow{2}{*}{ ICC } & \multicolumn{2}{|c|}{$95 \% \mathrm{CI}$} & \multicolumn{4}{|c|}{ F test with true value } \\
\hline & & Lower bound & Upper bound & Value & df1 & df2 & p-value \\
\hline Single & 0.980 & 0.955 & 0.991 & 93.773 & 25 & 25 & 0.000 \\
\hline Average & 0.990 & 0.977 & 0.995 & 93.773 & 25 & 25 & 0.000 \\
\hline
\end{tabular}

ICC: intraclass correlation coefficient, PCA: posterior condylar angle, CI: confidence interval.

The results of ICC analysis of the K-PACS measured PCA and the PCAs measured by our methods are summarized in Tables 2 and 3; there were statistically significant correlations $(\mathrm{p}=0.000)$ between PCAs measured by the proposed methods and by $\mathrm{K}$ PACS. Thus, one can infer that the angles measured by our methods correlate well with that measured by the CT-based K-PACS.

\section{Discussion}

Restoration of axial rotation of the femoral component in relation to TEA is a critical prerequisite for normal patellofemoral and tibiofemoral kinematics and balanced flexion gap in $\mathrm{TKA}^{9,12-19)}$. However, restoration of accurate rotation of the femoral component has been a challenge and failure to do so may to lead to patient dissatisfaction ${ }^{7,9,13,20,21)}$.

Despite the recognition of the importance of rotational alignment restoration, there is a paucity of methods which could be used during surgery for this concern. The available methods for estimating the rotational position of the femoral component during surgery rely on secondary reference axes ${ }^{14,16,22)}$. Geiger and Parsch $^{10)}$ used intraoperative CT scan for intraoperative determination of the correct rotational alignment before the implantation. They reported satisfactory results by use of intraoperative CT scan, but the cost, extra surgical time and radiation exposure need to be taken into consideration before routine employment of such a method.

Preoperative CT scan has been considered as gold standard to identify the TEA ${ }^{1)}$, but the same may not be replicated intraoperatively. In addition, it may not be possible and economically viable to get a preoperative CT scan for every case for PCA evaluation. Moreover, the preoperatively determined axis based on the CT scan may not accurately be reproduced intraoperatively as was shown by van der Linden-van der Zwaag et al. ${ }^{23)}$ in their CT-based study on rotational alignment accuracy of the femoral component in TKA using computer-assisted orthopaedic surgery. One reason for this discrepancy might be the fact that preoperative CT scan does not take into account the thickness of the cartilage on the condyles ${ }^{11)}$. We think that a method that allows for intraoperative measurement of the axis is better suited to take this factor into account.

For intraoperative application of the methods proposed by us, one would need a trained assistant capable of drawing a quadrilateral geometric figure based on the measurements provided by the surgeon and measuring the angle between lines using goniometer/protractor or trigonometric method with a scientific calculator which is available in all computers as well as smart phones. As per our proposed method, during surgery, the knee is flexed to $90^{\circ}$ after the distal femoral cut and the operating surgeon then identifies the medial and lateral epicondyles and marks both points with a sterile marker pen. A ruler is placed tangential to the posterior aspects of the two condyles; this represents the PCL. The surgeon then places another ruler on the medial epicondyle mark and drops it perpendicular to the first ruler. This corresponds to ML of the quadrilateral. Similar procedure is repeated on the lateral side to obtain the LL. The two marks on the first ruler (PCL) thus act as a limb of the quadrilateral with the TEA forming one of the other limbs of the quadrilateral. All the four lines described by us are then measured. One thus gets the measurements of all the four sides of the quadrilateral as in Fig. 4. Now the assistant has to draw the quadrilateral based on these measurements on a paper; most often it is trapezoidal in shape unless the PCL and TEA are parallel. After drawing the quadri- 


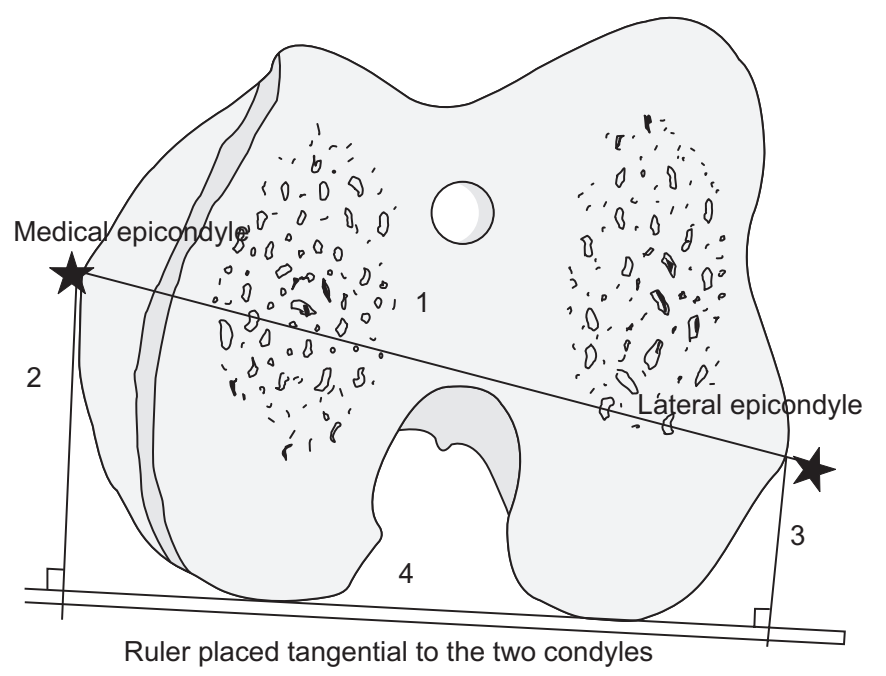

Fig. 4. Intraoperative measurements (1-4) after distal femoral cut using our methods.

lateral, methods 1 and 2 described previously can be applied.

We have proposed methods to assess the PCA intraoperatively such that it could be measured in real time without need for many instruments. In the present study, we applied the methods on CT scan image and evaluated the accuracy of each method in reference to the gold standard. We made an effort to statistically validate the accuracy of manual measurements with the software based measurements. All of the three methods resulted in similar angles and the mean angle measured by one method was not found to be statistically significantly different from that measured by the other method. Based on the statistical analysis, one can infer that trigonometrically and protractor measured PCA are equally accurate as the K-PACS measured PCA using CT scan. Importantly, the angles measured by our methods correlate well with that measured by the K-PACS.

As regards the actual utility of the study, it needs to be highlighted that we have put forward a method to measure PCA accurately and easily so that needful modification can be made while using posterior condylar axis as reference for flexion resection. The major limitation of the study is that we did not conduct this study on patients and just proposed methods. As the angles measured by K-PACS and our methods correlate well, we consider that these methods would actually be useful in real life. However, prior to application of these methods, they need to be validated by actual intraoperative studies or cadaveric studies. One more point of contention may be the abnormality in the shape of the condyles secondary to pathologies like osteoarthritis and rheumatoid arthritis which may give different readings on the CT scan and on the table during TKA. The very fact that cartilage thickness that CT scan does not take into consideration is reflected by our methods gives some theoretical advantage to our methods. The other limitation is the wide interobserver variability in the identification of TEA as was stated by Berger et al. ${ }^{1)}$ in 1993; however, in contradiction, a recent report by Oussedik et al. ${ }^{24)}$ in 2013 found more familiarity and accuracy in TEA identification.

\section{Conclusions}

In conclusion, we introduced simple, easily reproducible, realtime and radiation-free methods to measure the PCA during TKA. These methods can obviate the need for obtaining a preoperative CT scan for identification of patient specific PCA.

\section{Conflict of Interest}

No potential conflict of interest relevant to this article was reported.

\section{References}

1. Berger RA, Rubash HE, Seel MJ, Thompson WH, Crossett LS. Determining the rotational alignment of the femoral component in total knee arthroplasty using the epicondylar axis. Clin Orthop Relat Res. 1993;(286):40-7.

2. Mantas JP, Bloebaum RD, Skedros JG, Hofmann AA. Implications of reference axes used for rotational alignment of the femoral component in primary and revision knee arthroplasty. J Arthroplasty. 1992;7:531-5.

3. Thienpont E, Fennema P, Price A. Can technology improve alignment during knee arthroplasty. Knee. 2013;20 Suppl 1:S21-8.

4. Gothesen O, Slover J, Havelin L, Askildsen JE, Malchau H, Furnes $\mathrm{O}$. An economic model to evaluate cost-effectiveness of computer assisted knee replacement surgery in Norway. BMC Musculoskelet Disord. 2013;14:202.

5. Song EK, Seon JK, Yim JH, Netravali NA, Bargar WL. Robotic-assisted TKA reduces postoperative alignment outliers and improves gap balance compared to conventional TKA. Clin Orthop Relat Res. 2013;471:118-26.

6. Heesterbeek PJ, Jacobs WC, Wymenga AB. Effects of the balanced gap technique on femoral component rotation in TKA. Clin Orthop Relat Res. 2009;467:1015-22.

7. Chon JG, Sun DH, Jung JY, Kim TI, Jang SW. Rotational alignment of femoral component for minimal medial col- 
lateral ligament release in total knee arthroplasty. Knee Surg Relat Res. 2011;23:153-8.

8. Fitzgerald SJ, Trousdale RT. Why knees fail in 2011: patient, surgeon, or device? Orthopedics. 2011;34:e513-5.

9. Fehring TK. Rotational malalignment of the femoral component in total knee arthroplasty. Clin Orthop Relat Res. 2000;(380):72-9.

10. Geiger F, Parsch D. Intraoperative assessment of femoral component rotational alignment in total knee arthroplasty. Arch Orthop Trauma Surg. 2008;128:267-70.

11. Asada S, Akagi M, Matsushita T, Hashimoto K, Mori S, Hamanishi C. Effects of cartilage remnants of the posterior femoral condyles on femoral component rotation in varus knee osteoarthritis. Knee. 2012;19:185-9.

12. Stiehl JB, Abbott BD. Morphology of the transepicondylar axis and its application in primary and revision total knee arthroplasty. J Arthroplasty. 1995;10:785-9.

13. Olcott CW, Scott RD. A comparison of 4 intraoperative methods to determine femoral component rotation during total knee arthroplasty. J Arthroplasty. 2000;15:22-6.

14. Victor J. Rotational alignment of the distal femur: a literature review. Orthop Traumatol Surg Res. 2009;95:365-72.

15. Yoshino N, Takai S, Ohtsuki Y, Hirasawa Y. Computed tomography measurement of the surgical and clinical transepicondylar axis of the distal femur in osteoarthritic knees. J Arthroplasty. 2001;16:493-7.

16. Poilvache PL, Insall JN, Scuderi GR, Font-Rodriguez DE. Rotational landmarks and sizing of the distal femur in total knee arthroplasty. Clin Orthop Relat Res. 1996;(331):35-46.

17. Hollister AM, Jatana S, Singh AK, Sullivan WW, Lupichuk
AG. The axes of rotation of the knee. Clin Orthop Relat Res. 1993;(290):259-68.

18. Nordin M, Frankel VH. Biomechanics of the knee. In: Nordin M, Frankel VH, eds. Basic biomechanics of the musculoskeletal syetem. 2nd ed. Philadelphia, PA: Lea \& Febiger; 1989. p115-33.

19. Oswald MH, Jakob RP, Schneider E, Hoogewoud HM. Radiological analysis of normal axial alignment of femur and tibia in view of total knee arthroplasty. J Arthroplasty. 1993; 8:419-26.

20. Barrack RL, Schrader T, Bertot AJ, Wolfe MW, Myers L. Component rotation and anterior knee pain after total knee arthroplasty. Clin Orthop Relat Res. 2001;(392):46-55.

21. Matsuda S, Miura H, Nagamine R, Urabe K, Hirata G, Iwamoto Y. Effect of femoral and tibial component position on patellar tracking following total knee arthroplasty: 10-year follow-up of Miller-Galante I knees. Am J Knee Surg. 2001; 14:152-6.

22. Winemaker MJ. Perfect balance in total knee arthroplasty: the elusive compromise. J Arthroplasty. 2002;17:2-10.

23. van der Linden-van der Zwaag HM, Bos J, van der Heide HJ, Nelissen RG. A computed tomography based study on rotational alignment accuracy of the femoral component in total knee arthroplasty using computer-assisted orthopaedic surgery. Int Orthop. 2011;35:845-50.

24. Oussedik S, Scholes C, Ferguson D, Roe J, Parker D. Is femoral component rotation in a TKA reliably guided by the functional flexion axis? Clin Orthop Relat Res. 2012;470: 3227-32. 